\title{
Promoção da Saúde na Escola: história e perspectivas
}

\section{Health promotion in school: history and prospects}

\author{
Andréa Monteiro de Castro Graciano ${ }^{1}$, Natália Mendes Matos Cardoso ${ }^{2}$, Flávio Freitas Mattos $^{3}$, Viviane Elisângela Gomes ${ }^{3}$, \\ Ana Cristina Borges-Oliveira ${ }^{3}$
}

1. Doutora em Odontologia pela Faculdade de Odontologia da Universidade Federal de Minas Gerais (UFMG), Brasil. 2. Mestranda em Odontologia pela Faculdade de Odontologia da Universidade Federal de Minas Gerais (UFMG), Brasil. 3. Departamento de Odontologia Social e Preventiva da Faculdade de Odontologia da Universidade Federal de Minas Gerais (UFMG), Brasil.

\section{Resumo}

Introdução: A promoção da saúde nas escolas envolve a educação em saúde, a criação de entornos saudáveis e a provisão de serviços de saúde. Este estudo objetivou realizar uma revisão crítica da literatura sobre a promoção da saúde nas escolas. Metodologia: Os critérios prévios de inclusão foram: artigos que relacionavam saúde escolar e promoção da saúde, sem limite temporal de publicação. Foram realizadas buscas eletrônicas nas principais bases de dados, entre janeiro e março de 2014. Foram utilizadas as seguintes palavras-chave e conjunto de palavras: promoção da saúde, saúde escolar, health promotion e school health [termo MeSH e termo palavra]. Também foram pesquisados estudos que preencheram os critérios de inclusão originados da lista de referências dos artigos selecionados. Revisão de literatura/Discussão: A saúde escolar avançou em sintonia com o conhecimento técnico-científico e com o desenvolvimento sócio-político, superando, de forma gradativa, o paradigma biomédico para a concepção da iniciativa das Escolas Promotoras de Saúde. No Brasil, foi instituído um novo programa para a saúde do escolar denominado Programa Saúde na Escola (PSE). O PSE se identifica como uma estratégia para a integração e articulação permanente entre as políticas e ações de educação e saúde. Considerações finais: Uma escola promotora de saúde caracteriza-se como uma escola que busca um estilo de vida, aprendizagem e trabalho que favoreça o desenvolvimento da saúde.

Palavras-chave: Promoção da Saúde. Educação em Saúde. Saúde Escolar.

\section{Abstract}

Introduction: Health promotion in schools involves education on integral health, the creation of health surroundings and the provision of health services. This study aimed to conduct a critical review of literature about health promotion in school. Methodology: The previous inclusion criteria for the study were: articles that related school health and health promotion without the setting of a publishing time. Electronic searches on the main database, were carried out from January to March 2014. The following key words and combinations have been used: health promotion, school health (MeSH and word). The studies that met the criteria of inclusion from the references list of the selected articles have also been researched. Literature review/Discussion: School health has progressed in line with technical scientific knowledge and with the sociopolitical development, overcoming gradually the biomedical paradigm for the conception of the initiative of the Health Promoting Schools. In this context, with a new proposal in line with SUS and MEC it was implemented a new program for school health in the country called School Health Program (SHP). This program identifies itself as a strategy for the permanent integration and articulation among the policies and the actions of health and education. Final considerations: The articulation proposed by the SHP between education and health sectors may be considered an advance in view of the relevance of the intersectorial actions for the health promotion. The SHP must considerate the social and school contexts, the local diagnosis in the students' health and the operational capacity in the students' health.

Keywords: Health Promotion. Health Education. School Health.

\section{INTRODUCTION}

Considerando-se a evolução histórica do espaço escolar como um campo da promoção da saúde, nos últimos 30 anos, graças ao fortalecimento da democracia no Brasil e à luta pela cidadania, o trabalho educativo em saúde, vivenciado na escola, avançou muito no País. Este avanço viabilizou a inclusão de práticas educativas em saúde, no cotidiano didáticopedagógico das escolas. Além disso, contribuiu para uma maior consolidação da cooperação técnica entre os Ministérios da Saúde e da Educação, aumentando, dessa forma, a articulação entre a saúde e a educação nos espaços institucionais ${ }^{1}$.

No desenvolvimento de estratégias para a promoção da saúde, alguns aspectos pedagógicos da educação em saúde, como o desenvolvimento de uma visão crítica, são desafios práticos, que envolvem a problematização do cuidado, a aprendizagem significativa, a construção de conhecimento emancipatório, a autonomia nos cuidados com a saúde, assim como o direito à informação e à cidadania.2-4 A promoção da saúde nas escolas envolve a educação em saúde integral, a criação de entornos saudáveis e a provisão de serviços de saúde ${ }^{1,5}$.

Nos Parâmetros Curriculares Nacionais (PCN) para o ensino fundamental, organizam-se conteúdos e orientações didáticas para as atividades integradas às áreas curriculares, aos temas transversais e ao cotidiano da vida escolar. O ensino da saúde é tomado como um desafio para a educação, em que se almeja uma aprendizagem transformadora de atitudes, para que os alunos possam desenvolver hábitos desencadeadores de uma vida saudável. Ao educar para a saúde de forma contextualizada, o professor contribui para a formação de cidadãos capazes de atuarem em favor da coletividade ${ }^{2,6}$.

Buscando contribuir para uma reflexão sobre os aspectos históricos e perspectivas direcionadas à saúde escolar, este estudo objetivou realizar uma revisão crítica de literatura sobre a promoção da saúde nas escolas. 


\section{MÉTODOS}

Para a identificação dos estudos incluídos, ou considerados nesta revisão, uma estratégia de pesquisa foi desenvolvida nas principais bases de dados eletrônicas. Procurou-se utilizar palavras-chave da lista de cabeçalhos de assuntos médicos e educativos. Os critérios prévios de inclusão para o estudo foram: artigos que relacionavam saúde escolar e promoção da saúde, sem limite temporal de publicação. Foram realizadas buscas eletrônicas nas bases de dados: Literatura Latino-Americana e do Caribe em Ciências da Saúde (LILACS), PubMed, Scientific Electronic Library Online (SciELO), The Cochrane Library, ISI Web of Science e Medical Literature Analysis and Retrieval System Online (MEDLINE), nos meses de janeiro a março de 2014.

As seguintes palavras-chave e combinações foram utilizadas na busca dos estudos: promoção da saúde, saúde escolar, health promotion e school health [termo MeSH e termo palavra]. Também foram pesquisados estudos que preencheram os critérios de inclusão originados da lista de referências dos artigos selecionados.

Foram identificados 232 artigos nos idiomas português, espanhol e inglês, Após a seleção por título e resumo foram selecionados 192 artigos para a leitura na íntegra. A partir deles foram utilizadas 30 referências.

Por se tratar de uma revisão crítica de literatura, este estudo possibilita uma visão geral para contato e conhecimento amplo sobre o tema de estudo. Entretanto, por não se tratar de uma revisão sistemática, não permite a avaliação ou comparação dos aspectos levantados de forma isolada e fora do contexto geral construído.

\section{REVISÃO DE LITERATURA}

\section{Aspectos históricos}

No Brasil, a partir da década de 30 , as atividades de educação sanitária passaram a ser realizadas nas campanhas sanitárias, nas escolas e nos centros de saúde, mas nesta época propagavam conteúdos de puericultura ainda com ênfase nos aspectos biológicos e na consciência sanitária individual. Em 1942, com a criação do Serviço Especial de Saúde Pública (SESP), começaram a serem introduzidas técnicas novas de educação de grupos e de desenvolvimento para organização assistencial às comunidades ${ }^{2,7}$.

No campo da odontologia, particularmente, com origem e financiamento do modelo assistencial norte americano, este modelo denominado sistema incremental, organizava a atenção à saúde exclusivamente para os escolares. A assistência, na maioria das vezes, se sobrepunha à prevenção e à educação em saúde. 0 sistema incremental perdurou por 50 anos como modelo hegemônico de organização do cuidado em saúde bucal.8 Somente a partir da criação do Sistema Único de Saúde (SUS) e da implantação do Programa Saúde da Família (PSF), seguida da incorporação das equipes de saúde bucal no PSF em 2000 é que o sistema de saúde alcançou uma concepção e prática mais voltadas para a promoção da saúde ${ }^{9-12}$.

A saúde escolar avançou em sintonia com o conhecimento técnico-científico e com o desenvolvimento sócio-político, superando, de forma gradativa, o paradigma biomédico para a concepção da iniciativa das Escolas Promotoras de Saúde ${ }^{5,13}$.

Mas, nas décadas de 80 e 90, os programas de saúde escolar na América Latina e Caribe ainda se caracterizavam pela ênfase nas práticas higienistas, na prevenção de doenças transmissíveis, no tratamento de doenças e nos exames de triagem. A partir de 1995, a Organização Pan Americana de Saúde (OPAS) orientou o desenvolvimento de iniciativas regionais com enfoque mais amplo, que transcendessem métodos tradicionais, baseandose na promoção da saúde ${ }^{13}$. Nesse aspecto, no Brasil, a lei no 9.394 estabeleceu as diretrizes e bases da educação nacional trazendo como princípios a vinculação entre a educação escolar e as práticas sociais. Os educadores foram incumbidos de colaborar com as atividades de articulação da escola com as famílias e a comunidade. Ao educando, propôs-se assegurar a formação comum indispensável para o exercício da cidadania ${ }^{14}$.

Desde o lançamento formal, em 1995, da iniciativa, Escolas Promotoras de Saúde, as unidades escolares demonstraram bastante potencialidade como comunidades saudáveis. Contribuíram para a conquista de objetivos comuns em diferentes setores sociais, principalmente saúde e educação ${ }^{13}$.

As Escolas Promotoras de Saúde buscam fortalecer a capacidade dos setores saúde e educação para promoverem a saúde, o bemestar e a qualidade de vida dos escolares, pais, professores e outros membros da comunidade ${ }^{13,15}$. A promoção da saúde nas escolas engloba a educação em saúde com enfoque integral, a criação de entornos saudáveis e a provisão de serviços de saúde. A saúde escolar tem, portanto, sua prática higienista e assistencialista questionada, podendo avançar e ampliar a sua concepção e prática com uma visão integral e interdisciplinar do ser humano ${ }^{13,15}$. Nesse contexto, tendo em vista os PCNs, a Saúde deve ser considerada um tema transversal dos currículos escolares ${ }^{6}$.

Como uma estratégia relevante de promoção da saúde no âmbito escolar as Escolas Promotoras de Saúde representam um mecanismo articulado de esforços e recursos multissetoriais, orientados para o melhoramento das condições de saúde e bem-estar. Possibilita a ampliação das oportunidades para um aprendizado de qualidade, bem como um desenvolvimento sustentável para todos os indivíduos das comunidades educativas. Busca desenvolver, por meio de atividades educativas, conhecimentos e habilidades para o autocuidado e a prevenção das condutas de risco. Ainda pode incentivar uma análise crítica, por parte da comunidade escolar, de seus valores, condutas, condição social e estilos de vida, fortalecendo as medidas que contribuem para a melhoria da saúde e do desenvolvimento humano. Estimulando dessa forma, a participação de todos os atores da comunidade escolar 
na tomada de decisões ${ }^{5,13,15}$.

Em 2007, foi criada no Brasil a Portaria Interministerial $n^{\circ} 15$, por meio da Câmara Intersetorial entre o Ministério da Educação (MEC) e o Ministério da Saúde (MS). Tal portaria objetivou a elaboração de diretrizes que subsidiariam a Política Nacional de Educação em Saúde na Escola, destacando a necessidade de estratégias intersetoriais de educação e Saúde ${ }^{16}$.

Naquele mesmo ano, foi instituído, pela Portaria Interministerial no 17/2007 e regulamentado pelo Decreto 7.083/10, o Programa Mais Educação.23 Referia-se a uma estratégia do Ministério da Educação para induzir a ampliação da jornada escolar e a organização curricular na perspectiva da educação integral. As escolas das redes públicas de ensino deveriam desenvolver acompanhamento pedagógico e atividades em educação ambiental, esporte e lazer, direitos humanos, cultura e artes, cultura digital, promoção da Saúde, comunicação e uso de mídias, ciências da natureza e educação econômica ${ }^{17}$.

Também foi lançado em 2007 o Programa Mais Saúde: direito de todos. Propõe metas e ações, distribuídas em eixos de intervenção, com o propósito de melhorar o acesso às ações e garantir serviços de qualidade. Algumas destas ações foram implementadas pelo Ministério da Saúde e pelas demais entidades gestoras do SUS: realização de concursos públicos, fortalecimento do Serviço de Atendimento Móvel de Urgência (SAMU), implementação de Unidades de Pronto Atendimento (UPAs), ampliação do programa Saúde na Família e investimentos relacionados à promoção da saúde ${ }^{18}$.

Dentro desse contexto, com uma proposta em consonância com o SUS e com o MEC, foi instituído um novo programa para a saúde do escolar no país denominado Programa de Saúde na Escola (PSE) ${ }^{19-20}$.

\section{Programa Saúde na Escola (PSE)}

O PSE se identifica como uma estratégia para a integração e articulação permanente entre as políticas e ações de educação e saúde. Corresponde a um trabalho conjunto entre os Ministérios da Saúde e da Educação preconizado pelo Decreto Federal no 6.286, de 5 de dezembro de $2007^{19-20}$. O programa preconiza projetos e estratégias adotados pelos territórios com vistas à articulação entre os setores saúde e educação.19-20 De acordo com o Ministério da Saúde o PSE contribui com o fortalecimento de ações na perspectiva do desenvolvimento integral, possibilitando que a comunidade escolar participe de programas e projetos que articulem saúde e educação, visando controlar as vulnerabilidades que comprometem o desenvolvimento dos escolares brasileiros ${ }^{20}$.

O PSE cresceu muito no Brasil desde a sua criação, em 2007. Naquela época, 1.941.763 escolares estavam cobertos pelo programa. Em 2009 este número alcançou 6.560 .649 alunos em todo o País ${ }^{21}$.

A inclusão das escolas no PSE é definida pelos Secretários
Estaduais e Municipais de Educação e de Saúde, que devem considerar as prioridades e as metas de atendimento do programa ${ }^{22}$. De acordo com os autores, a adesão ao PSE, é formalizada pelo Ministério da Saúde conforme os critérios do programa, que prioriza as escolas mais vulneráveis, considerando o Índice de Desenvolvimento da Educação Básica (IDEB), a cobertura das Equipes de Saúde da Família (ESF) e a participação no Programa Mais Educação 23-24.

O programa envolve a atuação das ESF, Equipes de Saúde Bucal e de toda a comunidade escolar. O PSE objetiva contribuir para a formação integral dos estudantes da rede pública de educação básica por meio de ações de prevenção, promoção e atenção à saúde, que devem acontecer de forma concomitante (Quadro $1)^{19,22}$.

Quadro 1. Ações do Programa Saúde na Escola

\begin{tabular}{|c|c|}
\hline 1. Avaliação clínica e nutricional & $\begin{array}{l}\text { 9. Prevenção e redução do consumo } \\
\text { do álcool }\end{array}$ \\
\hline $\begin{array}{l}\text { 2. Promoção da alimentação } \\
\text { saudável }\end{array}$ & 10. Prevenção do uso de drogas \\
\hline 3. Avaliação oftalmológica & $\begin{array}{l}\text { 11. Promoção da saúde sexual e da } \\
\text { saúde reprodutiva }\end{array}$ \\
\hline $\begin{array}{l}\text { 4. Avaliação da saúde e da higiene } \\
\text { bucal }\end{array}$ & $\begin{array}{l}\text { 12. Controle do tabagismo e de outros } \\
\text { fatores de risco de câncer }\end{array}$ \\
\hline 5. Avaliação auditiva & 13. Educação permanente em saúde \\
\hline 6. Avaliação psicossocial & 14. Atividade física e saúde \\
\hline $\begin{array}{l}\text { 7. Atualização e controle do } \\
\text { calendário vacinal }\end{array}$ & $\begin{array}{l}\text { 15. Promoção da cultura da prevenção } \\
\text { no âmbito escolar }\end{array}$ \\
\hline $\begin{array}{l}\text { 8. Redução da morbimortalidade } \\
\text { por acidentes e violências }\end{array}$ & $\begin{array}{l}\text { 16. Inclusão da temática educação em } \\
\text { saúde no projeto político pedagógico } \\
\text { escolar }\end{array}$ \\
\hline
\end{tabular}

Adaptado ${ }^{19-20}$

O PSE traz uma contribuição inovadora, cujo diferencial teórico e prático é o potencial das ações promotoras da saúde, da intersetorialidade e do envolvimento comunitário ${ }^{19-20}$. O programa contempla o contexto nacional da Política Nacional de Atenção Integral à Saúde de Adolescentes e Jovens e a Política Nacional de Promoção da Saúde, que têm por objetivo promover a qualidade de vida e reduzir a vulnerabilidade e o risco. Considera também a Lei Orgânica da Saúde, a Lei das Diretrizes e Bases da Educação Nacional e a Portaria no 1.190, de 04 de junho de 2009, que institui o Plano de Acesso ao Tratamento e Prevenção em Álcool e Drogas (PEAD) ${ }^{10,14,21}$.

\section{DISCUSSÃO}

O caráter transformador da educação pode influenciar a constituição de vários aspectos da subjetividade das pessoas, como valores, crenças, orientações religiosas, sexuais, morais sentimentos, escolhas e muitos outros ${ }^{25}$. Dimensões mais abrangentes podem vir a conferir uma função social à escola, uma vez que o processo educativo que ocorre no seu interior seja entendido não apenas na dimensão do ensino e da aprendizagem de conhecimentos, mas também a partir das dimensões política, econômica e cultural ${ }^{25}$.

A definição de ambiente da escola deve incluir a participação e 
responsabilização dos estudantes pela vida escolar, bem como a relação com os professores e colegas, e a continuidade entre a vida familiar e escolar. Devido ao longo tempo de permanência dos jovens no local, a escola deve ser considerada um cenário chave, para intervenções destinadas a promover o bem-estar dos estudantes ${ }^{26}$.

Uma escola promotora de saúde deve contribuir para o desenvolvimento da saúde e educação dos escolares e da comunidade em que se encontram. No entanto, conforme salientaram Faria e Carvalho (2004), a criação de uma escola promotora de saúde não é um processo fácil ${ }^{27}$. São vários os fatores que dificultam a sua implantação. Entre eles destacase a fraca participação do setor saúde, a ausência de cursos de formação para professores na área de saúde; o tempo escasso dos professores para se dedicarem aos projetos de educação em saúde e o pouco envolvimento dos pais nas tomadas de decisão da escola. Segundo Gonçalves et al. (2008), um desafio a ser considerado refere-se à necessidade de se realizar uma revisão das propostas curriculares dos centros de formação de educadores em saúde, independente de serem esses profissionais da saúde ou da educação ${ }^{28}$. Os autores ressaltam a importância de um trabalho de conscientização sobre o desenvolvimento de atividades articuladas que envolvam os temas transversais.

Conforme ressaltaram Ferreira et al. (2012), o PSE foi criado com o intuito de contribuir para a formação integral dos escolares da rede pública de educação básica por meio de ações de prevenção, promoção e atenção à saúde ${ }^{22}$. Dando sustentação teórica ao PSE, alguns estudos identificaram uma relação direta entre o ambiente escolar e o bem-estar, destacando que as meninas apresentaram uma percepção mais evidente do bemestar no ambiente escolar que os meninos ${ }^{26,29-31}$. A percepção que os adolescentes têm de bem-estar está relacionada à possibilidade de integrarem comportamentos de saúde ao estilo de vida. A percepção dos jovens e do bem-estar mostrouse mais relevante na presença de um ambiente positivo na escola ${ }^{26,29-31}$. Os estudos supracitados são importantes porque conseguem identificar o ambiente escolar que, quando positivo e encorajador, é capaz de influenciar positivamente no comportamento e saúde dos escolares.
O PSE deve ser implementado por meio da adesão dos Estados, do Distrito Federal e dos Municípios, abrangendo os objetivos e diretrizes do programa22.

Por meio da consciência crítica e da ampliação da liberdade, a escola deve identificar-se como um ambiente que promova a interação pessoal, criativa e autônoma direcionada para a educação, ação, participação e autonomia ${ }^{4,22}$. A escola deve exercer o papel de educar os estudantes por meio do desenvolvimento de habilidades cognitivas e transmissão do conhecimento, auxiliando na construção de suas vidas e desenvolvendo habilidades individuais e sociais ${ }^{4,16,22}$. Tendo em vista que os comportamentos relacionados à saúde de um indivíduo também são influenciados por valores culturais e por forças sociais oriundas da sociedade à qual ele pertence, o ambiente escolar pode significar mais um cenário de transformação social. Assim, faz-se necessária uma reflexão sobre a complexa atitude de ensinar e aprender e as diferenças entre as opções pedagógicas.

\section{CONSIDERAÇÕES FINAIS}

Informações relacionadas a determinados fatores de risco para doenças, o desenvolvimento de atitudes individuais que promovam a saúde e a conscientização sobre os fatores econômicos e ambientais da saúde e doença muitas vezes são capazes de contribuir para organização de atividades pedagógicas dirigidas a mudanças ambientais, econômicas e sociais que promovam condições favoráveis à saúde.

Ao longo dos anos, o ambiente escolar tem se apresentado com muitos significados quanto à sua real função social, missão e organização. Nos dias de hoje, a escola é um equipamento social em que são desenvolvidos processos de ensino e aprendizagem articulados às ações de diferentes naturezas, envolvendo o seu território e área de abrangência.

Uma escola promotora de saúde caracteriza-se como uma escola que busca um estilo de vida, aprendizagem e trabalho que favoreça o desenvolvimento da saúde. O planejamento do PSE deve considerar o contexto escolar e social, o diagnóstico local em saúde do escolar e a capacidade operativa em saúde do escolar.

\section{REFERÊNCIAS}

1. Ministério da Saúde (Brasil). Escolas promotoras de saúde: experiências do Brasil / Ministério da Saúde, Organização Pan-Americana da Saúde. Brasília: Ministério da Saúde, 2006 [acesso 2014 mar 04]. (Série Promoção da Saúde; $\mathrm{n}$ ㅇ 6). Disponível em:

http://www.cedaps.org.br/wp-content/uploads/2013/07/esc_prom_saude. pdf.

2.FFerriani MG. A inserção do enfermeiro na saúde escolar: análise crítica de uma experiência [tese]. Ribeirão Preto: Universidade de São Paulo; 1988.

3. Pauleto AR, Pereira MLT, Cyrino EG. Saúde bucal: uma revisão crítica sobre programações educativas para escolares. Cien Saude Coletiva [Internet]. 2004 fev [acesso 2014 mar 17]; 9(1):121-130. Disponível em: http://www.scielo.br/ pdf/csc/v9n1/19829.pdf. doi: http://dx.doi.org/10.1590/S1413-81232004000100012.

4. Meyer DEE, Mello DFCM, Valadão MM, Ayres JR. Você aprende. A gente ensina? Interrogando relações entre educação e saúde desde a perspectiva da vulnerabilidade. Cad Saúde Pública [Internet]. 2006 jun [acesso em 2014 fev 28]; 22(6):1335-1342.

Disponível em: http://www.scielo.br/pdf/csp/v22n6/22.pdf. doi: http://dx.doi. org/10.1590/S0102-311X2006000600022.

5. Figueiredo TAN, Machado VLT, Abreu MMS. A saúde na escola: um breve resgate histórico. Cien Saude Coletiva. [Internet]. 2010 jul/dez [acesso em 2014 fev 15]; 15(2):397-402. Disponível em: http://www.scielo.br/pdf/csc/v15n2/ v15n2a15.pdf. doi: http://dx.doi.org/10.1590/S1413-81232010000200015. 
6. Ministério da Educação (Brasil), Secretaria de Educação Fundamental. Parâmetros curriculares nacionais: introdução aos parâmetros curriculares nacionais. [Internet] Brasília: MEC/SEF, 1997 [acesso 2015 mar 04]. Disponível em: <http://portal.mec.gov.br/seb/arquivos/pdf/livro01.pdf>.

7. Silva CMC, Meneghim MC, Pereira AC, Mialhe FL. Educação em saúde: uma reflexão histórica de suas práticas. Cien Saude Coletiva [Internet]. 2010 ago [acesso 2014 fev 02]; 15(5):2539-2550. Disponível em: http://www.scielo.br/ scielo.php?script=sci_arttext\&pid=S1413-81232010000500028. doi: http://dx.doi.org/10.1590/S1413-81232004000100012.

8. Nickel DA, Lima FG, Silva BB. Modelos assistenciais em saúde bucal no Brasil. Cad Saúde Pública [Internet]. 2008 fev [acesso 2014 fev 28]; 24(2):241-246. Disponível em: http://www.scielo.br/pdf/csp/v24n2/01.pdf. doi: http://dx.doi.org/10.1590/S0102-311X2008000200002

9. Brasil. Constituição da República Federativa do Brasil de 1988 [Internet] Brasília: Senado Federal; 1988 [acesso 2014 mar 17]. Disponível em: <http:// www.planalto.gov.br/ccivil_03/constituicao/constituicao.htm>.

10. Brasil. Lei $\mathrm{n} 08.080$, de 19 de setembro de 1990 . Dispõe sobre as condições para a promoção, proteção e recuperação da saúde, a organização e o funcionamento dos serviços correspondentes e dá outras providências [Internet]. Diário Oficial [da] República Federativa do Brasil. 1990 set. 19 [acesso 2014 mar 04]. Disponível em: <http://www.planalto.gov.br/ccivil_03/ leis//8080.htm>.

11. Ministério da Saúde (Brasil), Secretaria de Assistência à Saúde, Coordenação de Saúde da Comunidade. Saúde da Família: uma estratégia para a reorientação do modelo assistencial [Internet]. Brasília: Ministério da Saúde. 1997 [acesso 2014 mar 17]. Disponível em: <http://bvsms.saude.gov.br/bvs/publicacoes/ cd09_16.pdf>.

12. Brasil. Ministério da Saúde. Portaria no 1444, de 28 de dezembro de 2000. Estabelece incentivo financeiro para a reorganização da atenção à saúde bucal prestada nos municípios por meio do Programa de Saúde da Família [Internet]. Diário Oficial [da] República Federativa do Brasil. 2000 dez. 28 [acesso 2014 mar 17]. Disponível em: < http://www.camara.gov.br/sileg/integras/142359.pdf>.

13. Organização Mundial de Saúde, Organização Pan-Americana de Saúde. Escolas promotoras de saúde: fortalecimento da iniciativa regional, estratégias e linhas de ação 2003-2012. Washington: OPAS; 2006.

14. Brasil. Lei no 9.394, de 20 de dezembro de 1996. Estabelece as diretrizes e bases da educação nacional [Internet]. Diário Oficial [da] República Federativa do Brasil. 1996 dez 20 [acesso 2014 mar 04]. Disponível em: http://www. planalto.gov.br/ccivil_03/leis/19394.htm.

15. Chelala CA. Schools promoting health: healthy environments for better health in future generations. Washington: Organización Panamericana de la Salud. 1998. 32 p.

16. Brasil. Ministério da Educação. Portaria Normativa Interministerial no 15, de 24 de abril de 2007. Institui o "Projeto Olhar Brasil” [Internet]. Diário Oficial [da] República Federativa do Brasil. 2007 abr 24 [acesso 2014 mar 04]. Disponível em: <http://www.brasilsus.com.br/legislacoes/inter-ministerial/16299-15. html>

17. Brasil. Decreto no 7.083, de 27 de janeiro de 2010. Dispõe sobre o Programa Mais Educação [Internet]. Diário oficial [da] República Federativa do Brasil. 2010 jan 27 [acesso 2014 mar 04]. Disponível em: http://www.planalto.gov.br/ ccivil_03/_ato2007-2010/2010/decreto/d7083.htm.

18. Ministério do Planejamento (Brasil). Programa de aceleração do crescimento - 2007-2010. Brasília: Ministério do Planejamemto; 2007 [acesso 2014 mar 04]. Disponível em:<download.uol.com.br/fernandorodrigues/PAC?pac_ versaoparaimprensa.pd $>$.

19. Brasil. Decreto no 6.286, de 5 de dezembro de 2007. Institui o Programa Saúde na Escola - PSE, e dá outras providências [Internet]. Diário Oficial [da] República Federativa do Brasil. 2007 dez 05 [acesso 2014 mar 04]. Disponível em: <http:// portal.mec.gov.br/index.php?option=com_content\&id=14578\%3Aprogramasaude-nas-escolas\&Itemid=817>.

20. Ministério da Saúde (Brasil), Secretaria de Atenção à Saúde, Departamento de Atenção Básica. Passo a passo PSE. Programa Saúde na Escola: tecendo caminhos da intersetorialidade. Brasília: Ministério da Saúde; 2011 [acesso 2014 mar 28]. Disponível em: http://bvsms.saude.gov.br/bvs/publicacoes/ passo_a_passo_programa_saude_escola.pdf.

21. Brasil. Ministério da Saúde. Portaria no 1.190, de 04 de junho de 2009. Institui o Plano Emergencial de Ampliação do Acesso ao Tratamento e Prevenção em Álcool e outras Drogas no Sistema Único de Saúde - SUS (PEAD 2009-2010) e define suas diretrizes gerais, ações e metas. Diário Oficial [da] República Federativa do Brasil. 2009 jun 04 [acesso 2014 mar 04]. Disponível em: http:// bvsms.saude.gov.br/bvs/saudelegis/gm/2009/prt1190_04_06_2009.html.

22. Ferreira IRC, Vosgerau DSR, Moysés SJ, Moysés ST. Diplomas Normativos do Programa Saúde na Escola: análise de conteúdo associada à ferramenta Atlas TI. Cien Saude Coletiva. [Internet]. 2012 jul-dez [acesso em 2014 mar 12]; 17(12):3385-3398. Disponível em: http://www.scielo.br/pdf/csc/v17n12/23. pdf. doi: http://dx.doi.org/10.1590/S1413-81232012001200023.

23. Ministério da Educação (Brasil). Índice de Desenvolvimento da Educação Básica (IDEB) [Internet]. Brasília: MEC; 2007 [acesso 2014 mar 17]. Disponível em: <http://portal.mec.gov.br/index.php?option=com _ content\&view=article\&id=180\&ltemid=336\&msg=1>.

24. Ministério da Saúde (Brasil). Atenção Básica e a Saúde da Família [Internet]. Brasília: Ministério da Saúde; 2007 [acesso 2014 mar 17]. 2007d. Disponível em: <http://dab.saude.gov.br/atencaobasica.php\#saudedafamilia>.

25. Ministério da Justiça, Secretaria Nacional de Políticas sobre Drogas (Brasil). Curso de prevenção do uso de drogas para educadores de escolas públicas. 6 ed. Brasília: Ministério da Justiça. 2014. 272 p. [Acesso: 2014 março 04]. Disponível em: <http://educadores.senad.gov.br/images/Livro_texto_Cursode_ Prevencao_completo.pdf>.

26. Matos MG, Carvalhosa SF. A saúde dos adolescentes: ambiente escolar e bem-estar. Psic., Saúde \& Doenças [Internet]. 2001 nov [acesso em 2014 mar 10]; 2(2):43-53. Disponível em: http://www.scielo.gpeari.mctes.pt/pdf/psd/ v2n2/v2n2a03.pdf.

27. Faria HA, Carvalho GS. Escolas promotoras de saúde: factores críticos para o sucesso da parceria escola-centro de saúde. Rev Portuguesa de Saúde Pública [Internet]. 2004 jul-dez [acesso 2014 jan 14]; 22(2):79-90. Disponível em: https://www.ensp.unl.pt/dispositivos-de-apoio/cdi/cdi/sector-de-publicacoes/ revista/2000-2008/pdfs/2-07-2004.pdf.

28. Gonçalves FD, Catrib AMF, Vieira NFC, Vieira LJES. A promoção da saúde na educação infantil. Interface [Internet]. 2008 jan-mar [acesso 2014 fev 15]; 12(24):181-192. Disponível em: http://www.scielo.br/pdf/icse/v12n24/13.pdf. doi: http://dx.doi.org/10.1590/S1414-32832008000100014.

29. Nutbeam D, Smith C, Moore L, Bauman A. Warning! School can damage your health: alienation from school and its impact on health behaviour. J Paediatr Child Health. 1993; 29(Suppl. 1):S25-S30. PubMed PMID: 8268018.

30. Samdal O, Nutbeam D, Wold B, Kannas L. Achieving health and educational goals through schools: a study of the importance of the school climate and the students' satisfaction with school. Health Educ Res. 1998 fev [acesso em 2014 fev 10]; 13(3):383-397. Disponível em: http://her.oxfordjournals.org/ content/13/3/383.full.pdf+html.

31. Battistich $V$, Hom $A$. The relationship between students' sense of their school as a community and their involvement in problem behaviors. Am J Public Health. 1997 Dec; 87(12):1997-2001. PMCID: PMC 1381243.

\section{Como citar este artigo/How to cite this article:}

Graciano AMC, Cardoso NMM, Mattos FF, Gomes VE, Borges-Oliveira AC. Promoção da Saúde na Escola: história e perspectivas. J Health Biol Sci. 2015 JanMar; 3(1): 34-38 